Journal of Animal and Veterinary Advances 11 (10): 1633-1642, 2012

ISSN: $1680-5593$

(C) Medwell Journals, 2012

\title{
Mid-Field Magnetic Resonance Imaging and Cross-Sectional Anatomy of the Yearling Chinese Equine Distal Forelimb
}

\author{
${ }^{1}$ Xing Lv, ${ }^{3}$ Haibin Zhu, ${ }^{4}$ Xiang Gao, ${ }^{1}$ Xiaojian Song, ${ }^{2}$ Yuanhui Ma, \\ ${ }^{3}$ Xiaoying Wang, ${ }^{1}$ Jing Fang and ${ }^{1}$ Jue Zhang \\ ${ }^{1}$ Department of Biomedical Engineering, College of Engineering, \\ ${ }^{2}$ College of Life Sciences, Peking University, Beijing, China \\ ${ }^{3}$ Department of Radiology, Peking University First Hospital, Beijing, China \\ ${ }^{4}$ China Modern Pentathlon and Equestrian Training Base, Beijing, China
}

\begin{abstract}
The objective of this study is to perform mid-field (0.4T) Magnetic Resonant Imaging (MRI) on a distal forelimb of a healthy Mongolian yearling horse and obtain a detailed cross-sectional comparison between the MRI and anatomy images. T1-weighted, T2-weighted, PD-weighted and STIR sequence were performed to acquire images of the horse distal limb on the transverse plane. After imaging, the limb was frozen and cut into slices to get anatomy photos. Comparisons between the MR images and corresponding frozen cross-sections of the horse limb were made to identify the anatomic details of the horse limb. The results of this study indicate that mid-field MRI can be used to diagnose lesions in the equine distal forelimb at an early stage of lameness. Besides, this study also provides an atlas of gross anatomy of the carpus and fetlock in mid-field MRI which may benefit veterinary clinical evaluation and medical research.
\end{abstract}

Key words: Mid-field MRI, equine, distal limb, anatomy, forlimb, horse

\section{INTRODUCTION}

Lameness localized in the carpus and fetlock region has been the most frequently identified chronic, low-grade lesions on forelimb in sport horses (Blaik et al., 2000). A major difficulty encountered in equine lameness diagnosis lies in the complex anatomy of the distal horse limb, especially the carpus with its multiple small bones, numerous soft tissue structures and synovia. Magnetic Resonance Imaging (MRI) has been increasingly used in the diagnosis of equine lameness recently. It offers a higher soft-tissue contrast andpermits a more direct evaluation of articular cartilage, tendons, ligaments, joint capsules and synovium andbone than other medical imaging techniques (Mair and Kinns, 2005; Bureau et al., 1995; Buckwalter, 1996; Widmer et al., 1999).

There have been many reports about the identification of lameness on equines using low-field and high-field MRI (Dyson and Murray, 2007; Murray et al., 2007; Branch et al., 2007; Straaten and Collins, 1995; Dyson and Murray, 2007). Low-field MRI image scanners with a magnetic field of $<0.3 \mathrm{~T}$ are popular for equine imaging due to their low cost and open bore design. However, they usually suffer from a low signal to noise ratio and are therefore of limited clinical significance. High-field MRI scanners with a magnetic field of $>1 \mathrm{~T}$, acquire images with better image quality within a shorter time in comparison to low-field MRI. They are particularly good at identifying early signs at the onset of the disease within the equine foot (Murray et al., 2004; Whitton et al., 1998; Parizel et al., 1995; Choquet et al., 2000).

However, high-field MRI systems are usually expensive and require high-level maintenance. Furthermore, most of the high-field hardware systems bear a bore super-conducting design which requires additional specific instruments for the fixation and movement of the equine limbs.

Mid-field MRI with a magnet filed between low and high filed MRI has been an alternative solution considering a compromise between the contrast to noise ratio and maintenance cost (Rutt and Lee, 1996). However, reports about the mid-field MRI used in equine studies have been scarce.

In this study, researchers investigated the performance of mid-field MRI in examining the crosssectional anatomy of the equine distal limb in transverse planes by using Proton Density (PD), Short Inversion

Corresponding Author: Jue Zhang, Department of Biomedical Engineering, Center for Functional Imaging, Peking University, Yiheyuan Road No. 5, 100871 Beijing, China 
Recovery (STIR), T1-weghted and T2-weighted imaging sequences. The aim of the study is to evaluate the potential of the mid-field MRI in lameness diagnosis through a comparison between the anatomical crosssectional slices of the whole equine distal forelimb and their MR images.

\section{MATERIALS AND METHODS}

One distal forelimb from a healthy Chinese Mongolian female yearling horse ( 1 year old) was studied. The limb was scanned $8 \mathrm{~h}$ after death. Before scanning, the limb was cut into four main parts: knee, cannon bone, fetlock and foot.

Each part was placed in an extended position within a specifically built homemade quadrature volume RF coil and imaged with a $0.4 \mathrm{~T}$ scanner (Taijie MRI, Beijing). Images were obtained in 3 planes with T1-weighted, T2weighted, Proton Density (PD) and Short Tau Inversion Recovery (STIR) sequences. The parameters of each sequence are shown in Table 1.

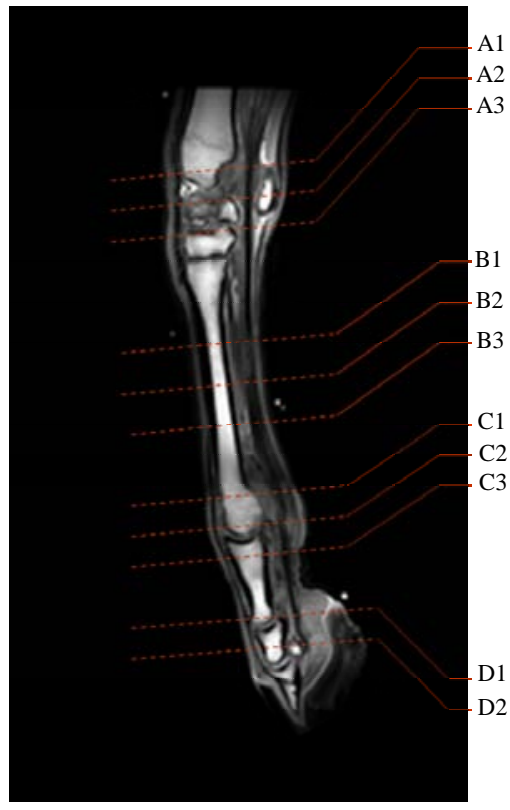

Fig. 1: The positions of slices in the forelimb

Table 1: Parameters of MRI sequence used for imaging

\begin{tabular}{|c|c|c|c|c|}
\hline \multirow[b]{2}{*}{$\underline{\text { Sequences }}$} & \multicolumn{4}{|c|}{ Parameters } \\
\hline & $\mathrm{TR}^{\mathrm{a}}$ & $\mathrm{TE}^{\mathrm{b}}$ & Flip angle $^{c}$ & $\begin{array}{c}\text { Slice } \\
\text { thickness }(\mathrm{mm})^{\mathrm{d}}\end{array}$ \\
\hline T1-weighted (SE) & 440 & 14.5 & 90 & 7 \\
\hline T2-weighted (FSE) & 4200 & 120.0 & 90 & 8 \\
\hline PD (FSE) & 3000 & 21.0 & 90 & 7 \\
\hline STIR (FSE) & 4800 & 84.0 & 90 & 7 \\
\hline
\end{tabular}

In order to make an accurate correlation between the anatomical structures and MRI images, glycerin capsule markers ( $5 \mathrm{~mm}$ in diameter and $20 \mathrm{~mm}$ in length) were attached to each limb joint before imaging which also acted as reference points for gross sectioning. After imaging, the limb was frozen at $-15^{\circ} \mathrm{C}$ in refrigerating room for $10 \mathrm{~h}$ and was then axially sectioned into slices of $5 \mathrm{~mm}$ thickness. The cross section of each slice was photographed with a digital camera (SONY, DSC-w220, $1536 \times 2048$ ) for comparison with its corresponding MR image.

The positions of slices are shown in Fig. 1 on the sagittal view of the forelimb which was generated by splicing the $\mathrm{T} 1$-weig hted plane images of four parts vertically without overlap (Photoshop 7.0, Corp. Adobe). A1-A3 represents the positions of the four slices in carpus; B1-B3 label the three slices in cannon; C1-C3 indicate the three slices in fetlock; D1-D2 point to the three slices in hoof.

\section{RESULTS AND DISCUSSION}

The findings of this study are organized as sequential pairs of anatomic slices and their corresponding MR images in transverse plane. Clinically relevant anatomic structures are identified and marked on both figures (MR image and anatomic section photograph). The T1-weighted images, T2-weighted images, PD images and STIR images of carpus, cannon bone, fetlock and hoof are shown in Fig. 2-12.

In the study, T1 and PD weighted images clearly presented the anatomical details of equine forelimb. Bony structures, especially these of the smaller tarsal bones andsoft tissue structures surrounding joints are well visualized in transverse plane. Tendons including the associated sheaths and their linkage with osseous structures, are clearly identified. In T1-weighted images, the deep digital flexor tendon are of low signal intensity and are coded in black or dark gray whereas the surrounding ligament, tendon, muscle and cartilage appear in bright white due to high signal intensities. The coded colors of normal tissues imaged with different sequence are shown in Table 2 .

Table 2: Coded colors of normal tissues imaged with different sequence

\begin{tabular}{|c|c|c|c|c|}
\hline Tissue & $\mathrm{T} 1^{\mathrm{a}}$ & $\mathrm{T} 2^{\mathrm{b}}$ & $\mathrm{PD}^{\mathrm{c}}$ & STIR $^{\mathrm{d}}$ \\
\hline Articular cartilage & White & Gray & White & White \\
\hline Cortical bone & Black & Black & Black & Black \\
\hline Bone marrow & White & White & White & Gray \\
\hline Muscle & $\begin{array}{l}\text { Dark gray } \\
\text { to black }\end{array}$ & Black & Black & Black \\
\hline Fat & White & White & Gray white & Gray black \\
\hline Synovium & Gray & White & Gray white & White \\
\hline Vessel & Gray & White & Gray & White \\
\hline Ligament/tendon & $\begin{array}{l}\text { Dark gray } \\
\text { to black }\end{array}$ & Black & $\begin{array}{l}\text { Dark gray } \\
\text { to black }\end{array}$ & Black \\
\hline
\end{tabular}

Tau Inversion Recovery 
(a)
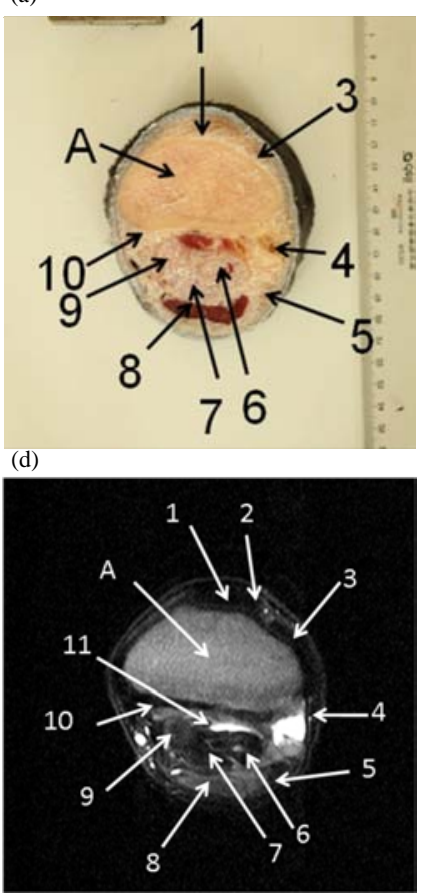

(b)
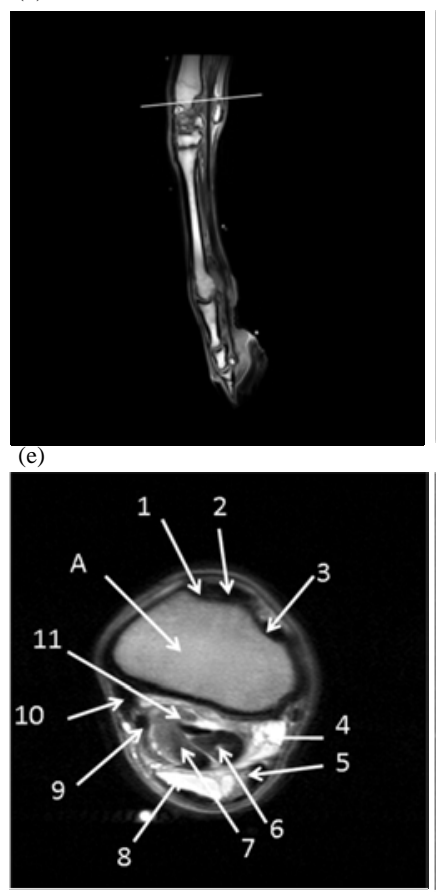

(c)

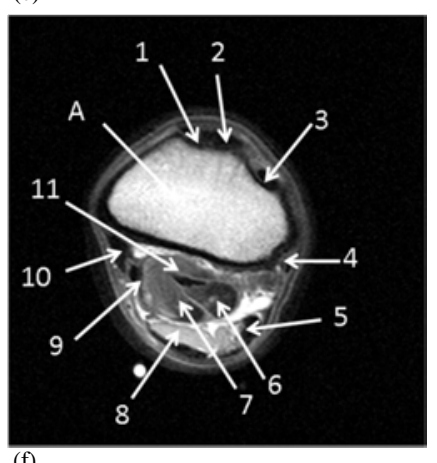

(f)

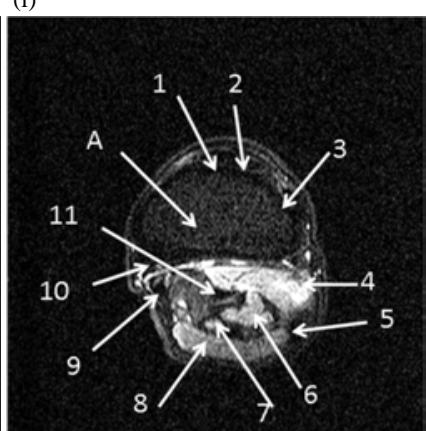

Fig. 2: a) The anatomic slice of carpus; b) The position of the slice in the forelimb; c) T1-weighted image in transverse plane; d) T2-weighted image in transverse plane; e) PD image in transverse plane and f) STIR image in transverse plane. A: Radius, 1: Extensor carpi radialis muscle, 2: Oblique carpal extensor muscle, 3: Common digital extensor muscle, 4: Lateral digital extensor muscle, 5: Ulnaris lateralis muscle, 6: Deep digital flexor tendon, 7: Superficial digital flexor tendon, 8: Flexor carpi ulnaris muscle, 9: Flexor carpi radialis muscle, 10: Medial collateral ligament of carpus joint, 11: Radial head of the deep digital flexor muscle

For equine lameness diagnosis in distal limbs, a significant advantage of MRI over other conventional imaging techniques is that it offers a simultaneous imaging of both bone and soft tissues with a high contrast. In addition, MRI allows the assessment of a variety of physiological differences between normal and abnormal tissues by employing a range of corresponding imaging sequences. For instance, the diagnosis of early stage chronic carpal arthritis such as osteoarthritis, synovitis and articular capsulitis by using X-ray or ultrasonograph is usually inconvenient due to the complicated structure of carpus (Fig. 2-4). Suspensory ligament, ligament inflammation, chronic tendonitis andaccessory ligament of the deep digital flexor tendon which usually occurs in cannon bone or in the internals of bones are difficult to locate with routine examinations (Fig. 5-7). In these areas, MRI can not only provide a clear separation between the bone and soft tissue but also has the potential to noninvasively pinpoint the location of the lesion point with a precision in $\mathrm{mm}$. The MR scanner we used should be categorized as a mid-field MRI, according to Rutt and Lee (1996) and Marti-Bonmati and Kormano (1997). Compared with high-field MRI, mid-field MRI with a permanent magnet design requires lower initial cost, lower operating costs and no cryogens. The limited fringe field also fits the anesthetic equipments in the scanning room. Besides, with the development of the hardware design of the MRI techniques and mid-field MRI could be easily designed in a moveable U-style or upside down U-style mode.

Therefore, the open design of the mid-field MRI is especially suitable for in vivo diagnosis of the distal limb of large standing animals like horses. Compared with lowfield MRI, mid-field MRI provides a better Signal-to-Noise Ratio (SNR) in T1-weighted, T2-weigthed and PDweighted images acquired within a shorter time.

The successful implementation of the STIR sequence on the equine fore limb is another advantage of this mid-field study. This application may benefit the clinical equine diagnosis and also provide a suitable method for diagnosing contusion located in limbs of equine. 
(a)
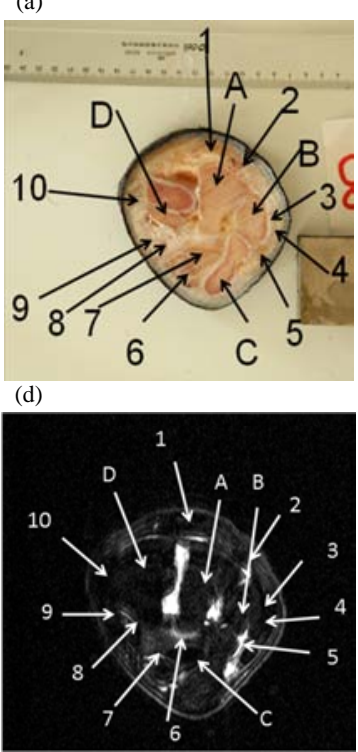

(b)

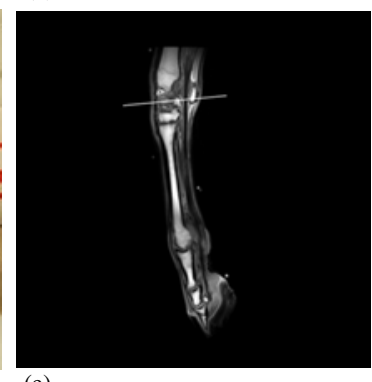

(e)

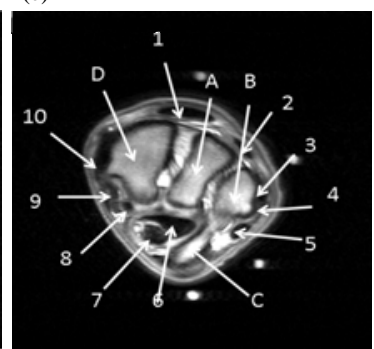

(c)

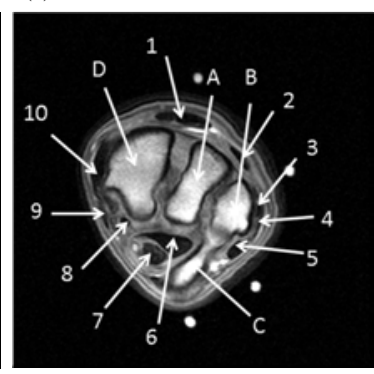

(f)

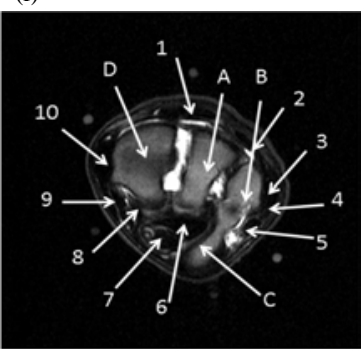

Fig. 3: a) The anatomic slice of carpus; b) The position of slice in the forelimb; c) T1-weighted image in transverse plane; d) T2-weighted image in transverse plane; e) PD image in transverse plane and f) STIR image in transverse plane. A: Intermediate carpal bone; B: Ulnar carpal bone; C: Pisiform bone; D: Radial carpal bone. 1: Extensor carpi radialis muscle; 2: Common digital extensor muscle; 3 : Lateral digital extensor muscle; 4: Lateral collateral ligament of carpus; 5: Tendon of ulnaris lateralis; 6: Deep digital flexor tendon; 7: Superficial digital flexor tendon; 8: Flexor carpi radialis muscle; 9: Carpal flexor retina culum; 10: Medial collateral ligament of carpus

(a)
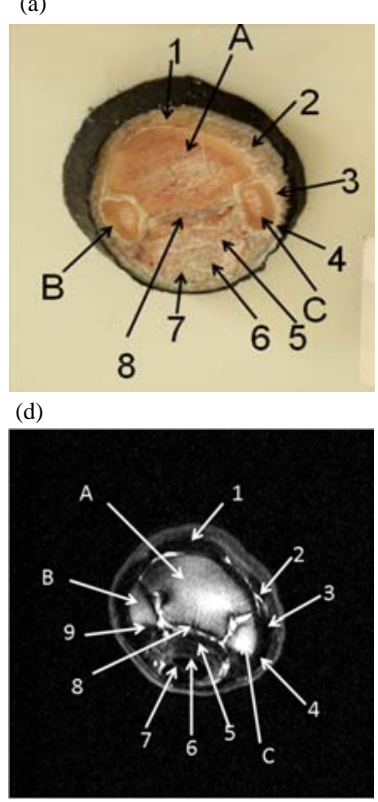

(b)

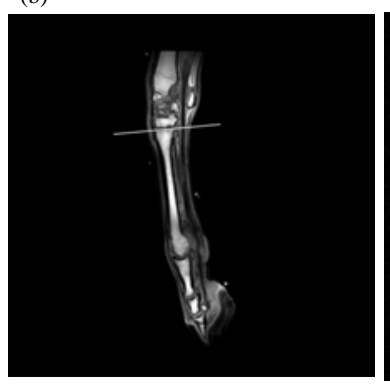

(e)

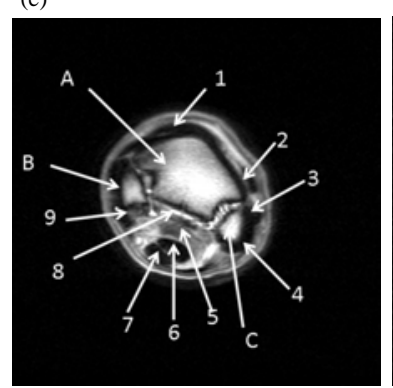

(c)

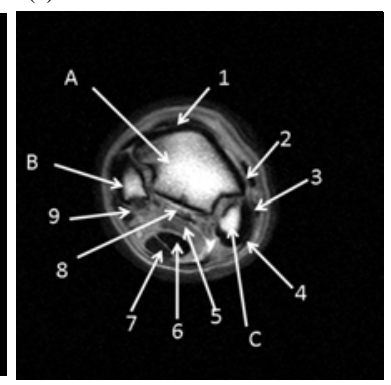

(f)

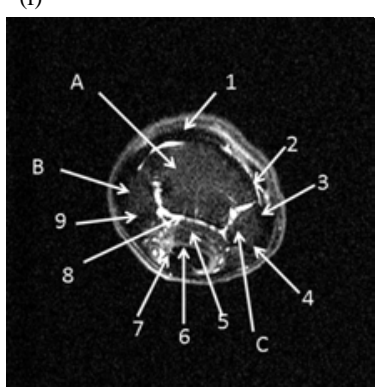

Fig. 4: a) Gross anatomic slice of carpus; b) the position of slice in the forelimb; c) T1-weighted image in transverse plane; d) T2-weighted image in transverse plane; e) PD image in transverse plane and f) STIR image in transverse plane. A: Cannon bone; B: Medial split bone; C: Lateral split bone; 1: Extensor carpi radialis muscle; 2: Common digital extensor muscle; 3: Lateral digital extensor muscle; 4: Lateral collateral ligament of carpus joint; 5: Distal check ligament; 6: Deep digital flexor tendon; 7: Superficial digital flexor tendon; 8: Inter osseous muscle; 9: Flexor carpi radialis muscle 
(a)

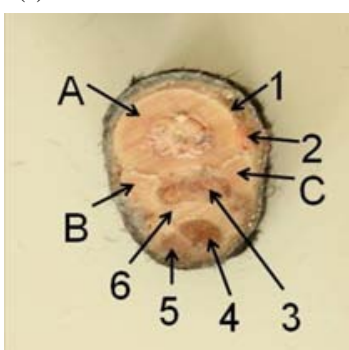

(d)

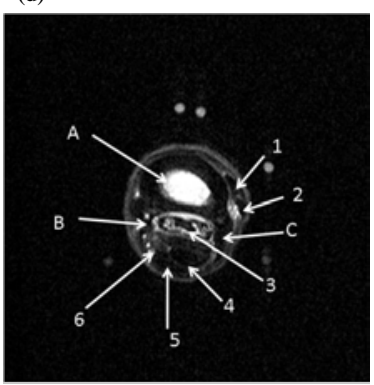

(b)

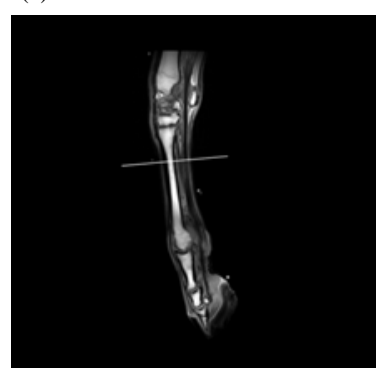

(e)

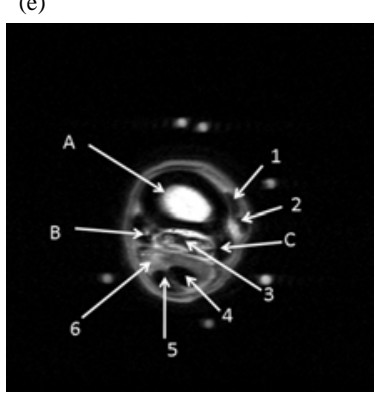

(c)

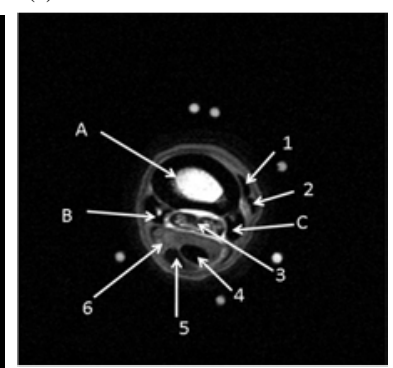

(f)

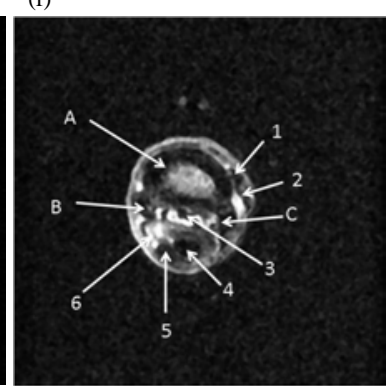

Fig. 5: a) Gross anatomic slice of cannon; b) the position of slice in the forelimb; c) T1-weighted image in transverse plane; d) T2-weighted image in transverse plane; e) PD image in transverse plane and f) STIR image in transverse plane. A: Cannon bone; B: Medial split bone; C: Lateral split bone; 1: Common digital extensor muscle; 2: Lateral digital extensor muscle; 3: Interosseous muscle; 4: Deep digital flexor tendon; 5: Superficial digital flexor tendon; 6: Distal check ligament

(a)

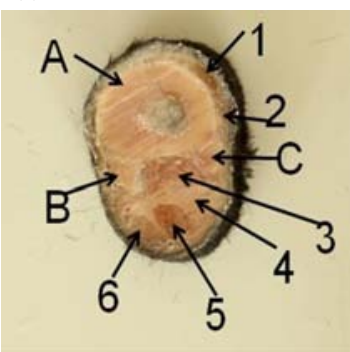

(d)

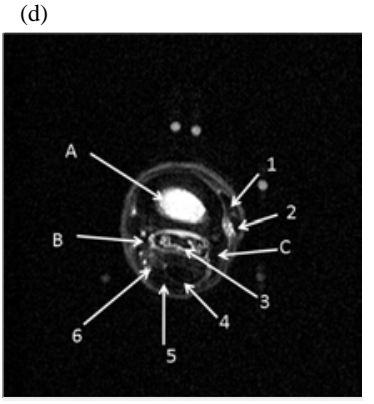

(b)

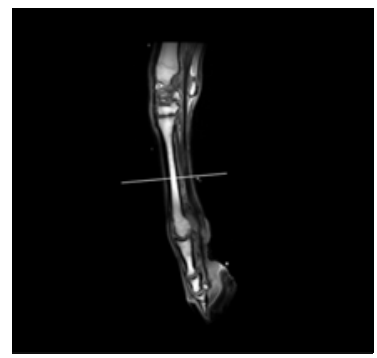

(e)

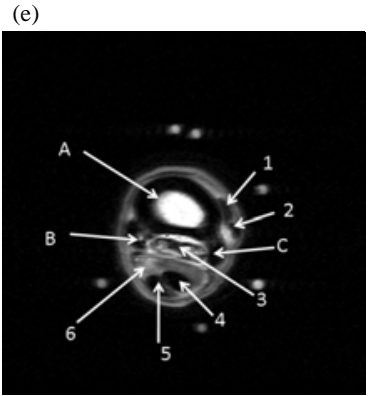

(c)

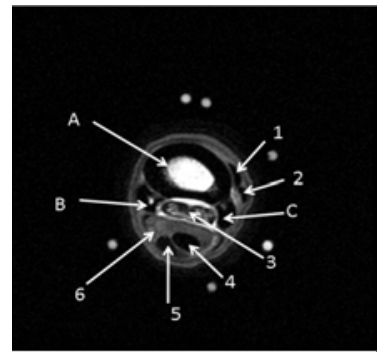

(f)

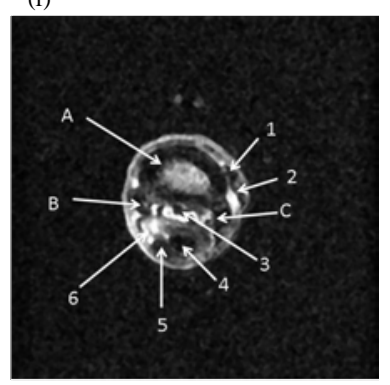

Fig. 6: a) Gross anatomic slice of cannon; b) the position of slice in the forelimb; c) T1-weighted image in transverse plane; d) T2-weighted image in transverse plane; e) PD image in transverse plane and f) STIR image in transverse plane. A: Cannon bone; B: Medial split bone; C: Lateral split bone. 1: Common digital extensor muscle; 2: lateral digital extensor muscle; 3: Interosseous muscle; 4: Distal check ligament; 5: Deep digital flexor tendon; 6: Superficial digital flexor tendon 
(a)

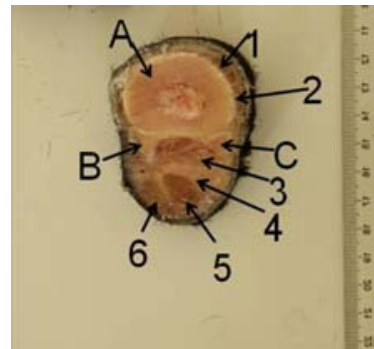

(d)

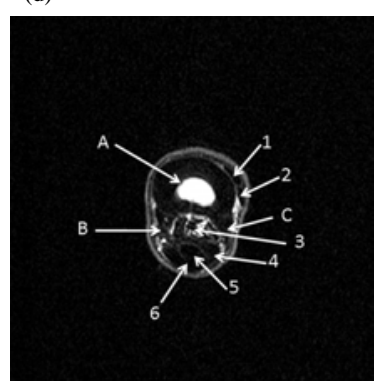

(b)

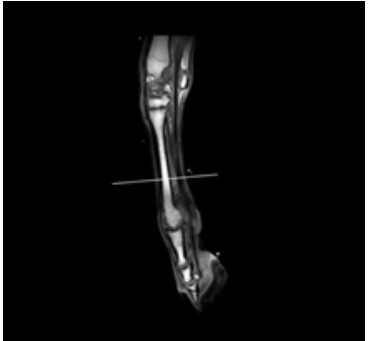

(e)

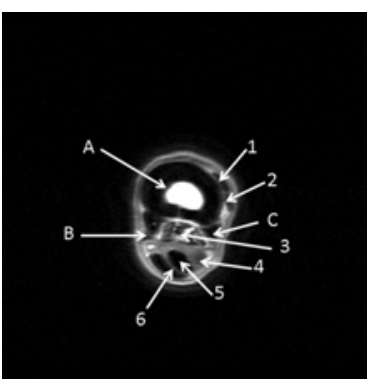

(c)

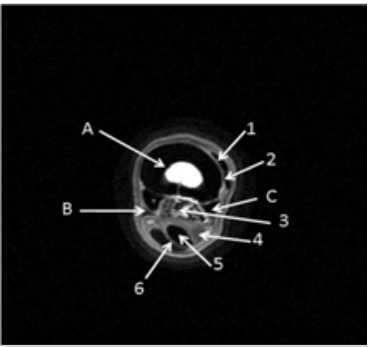

(f)

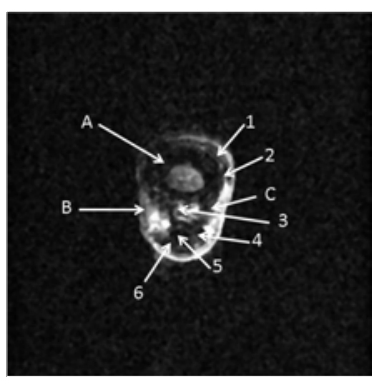

Fig. 7: a) Anatomic slice of cannon; b) the position of slice in the forelimb; c) T1-weighted image in transverse plane; d) T2-weighted image in transverse plane; e) PD image in transverse plane and f) STIR image in transverse plane. A: Cannon bone; B: Medial split bone; C: Lateral split bone. 1: Common digital extensor muscle; 2: lateral digital extensor muscle; 3: Interosseous muscle; 4: Distal check ligament; 5: Deep digital flexor tendon; 6: Superficial digital flexor tendon

(a)

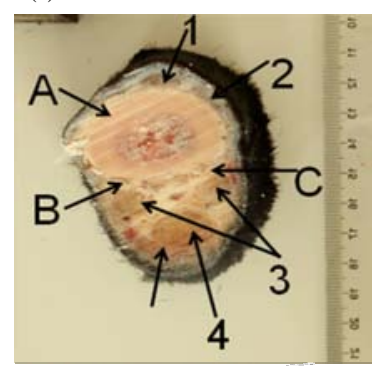

(d)

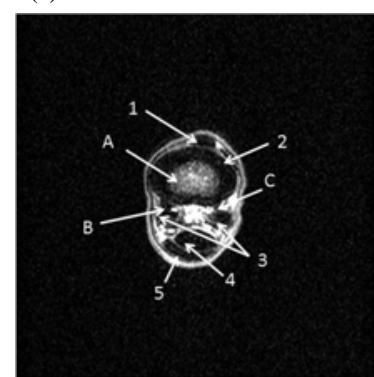

(b)

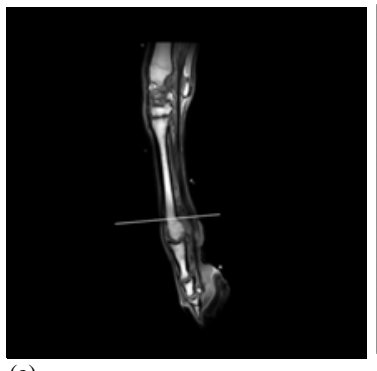

(e)

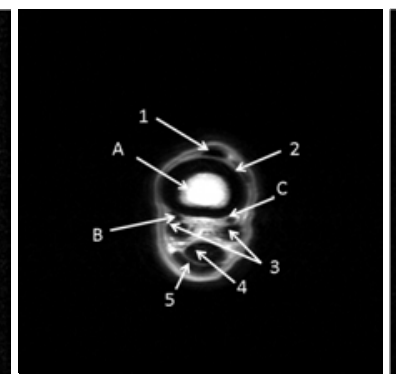

(c)

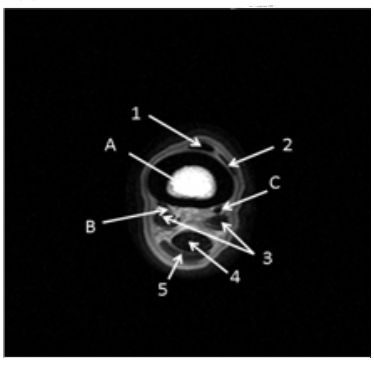

(f)

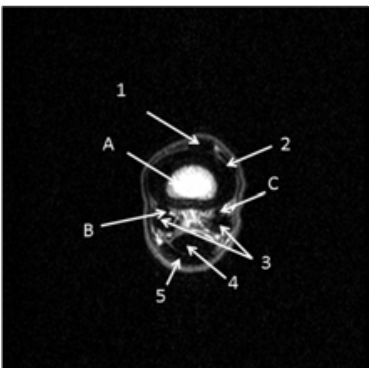

Fig. 8: a) Gross anatomic slice of fetlock; b) the position of slice in the forelimb; c) T1-weighted image in transverse plane; d) T2-weighted image in transverse plane; e) PD image in transverse plane and f) STIR image in transverse plane. A: Cannon bone; B: Medial split bone; C: Lateral split bone. 1: Common digital extensor muscle; 2: Lateral digital extensor muscle; 3: Branch of suspensory jointing extensor tendon; 4: Deep digital flexor tendon; 5: Superficial digital flexor tendon 
(a)

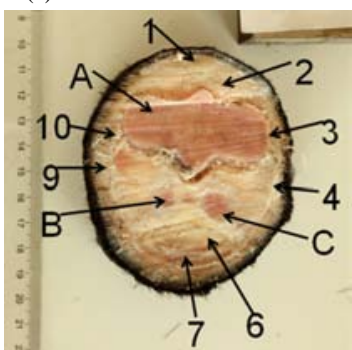

(d)

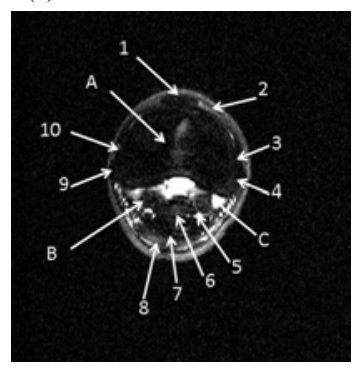

(b)

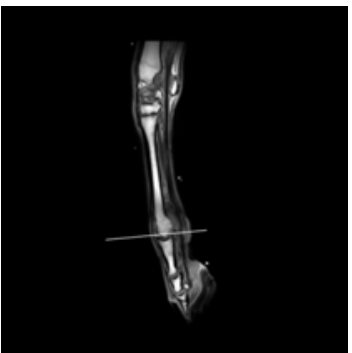

(e)

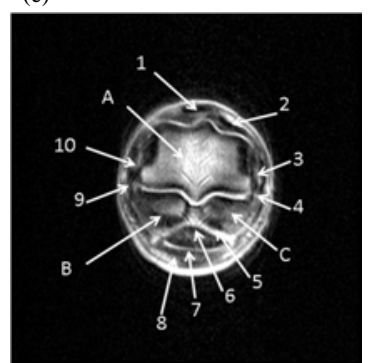

(c)

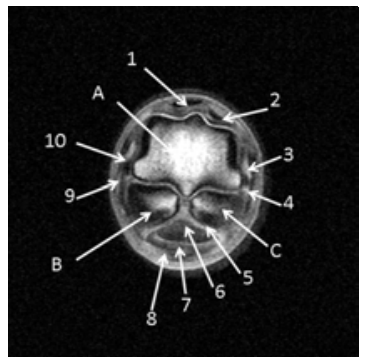

(f)

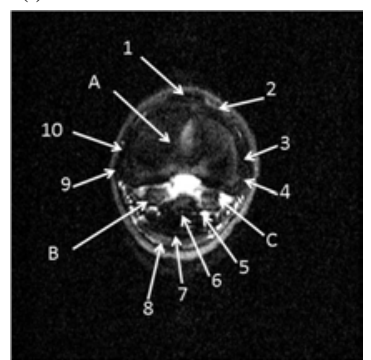

Fig. 9: a) The anatomic slice of fetlock; b) the position of slice in the forelimb; c) T1-weighted image in transverse plane; d) T2-weighted image in transverse plane; e) PD image in transverse plane and f) STIR image in transverse plane. A: Cannon bone B: Medial proximal sesamoid bone C: Lateral proximal sesamoid bone 1: Common digital extensor muscle; 2: Lateral digital extensor muscle; 3: Lateral collateral ligament of fetlock joint; 4: Lateral branch of suspensory jointing extensor tendon; 5 Digital flexor tendon sheath; 6: Deep digital flexor tendon; 7: Superficial digital flexor tendon; 8: Palmar annular ligament; 9: Medial branch of suspensory jointing extensor tendon; 10: Medial collateral ligament of fetlock joint

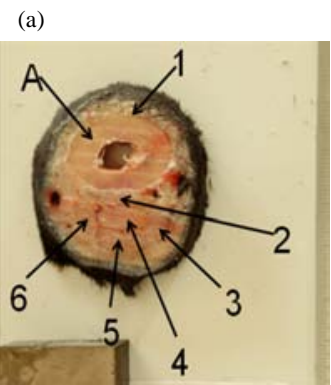

(d)

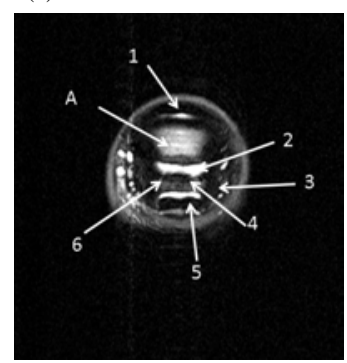

(b)
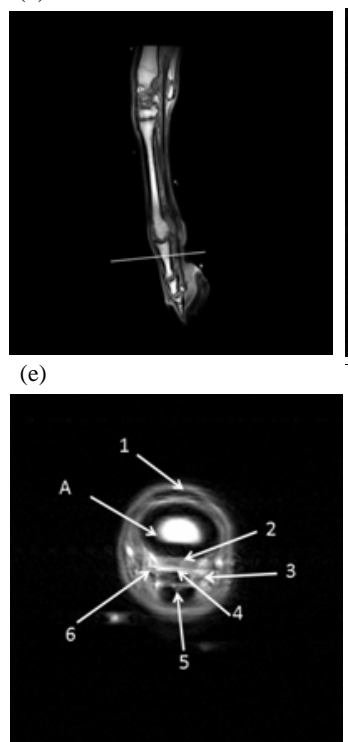

(c)
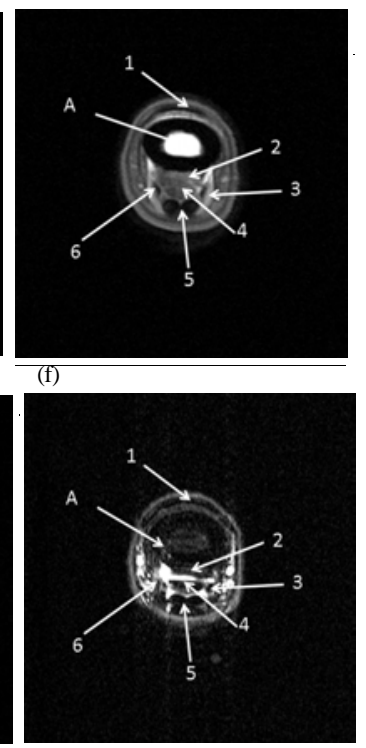

Fig. 10: a) Gross anatomic slice of fetlock; b) the position of slice in the forelimb; c) T1-weighted image in transverse plane; d) T2-weighted image in transverse plane; e) PD image in transverse plane and f) STIR image in transverse plane. A: Long pastern bone 1: Common digital extensor muscle; 2: Oblique sesamoid ligament; 3: Lateral aspect of superficial digital flexor; 4: Straight sesamoid ligament; 5: Deep digital flexor tendon; 6: Medial aspect of superficial digital flexor 
(a)

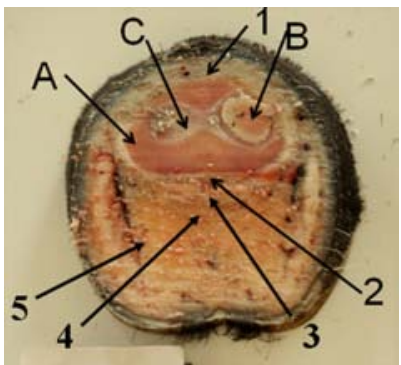

(d)

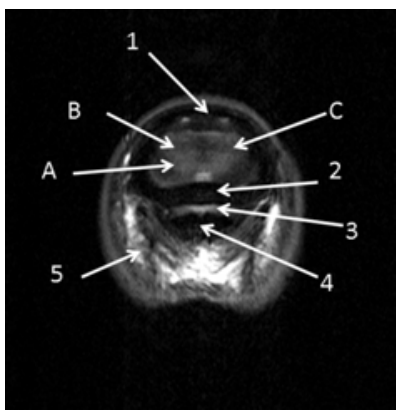

(b)

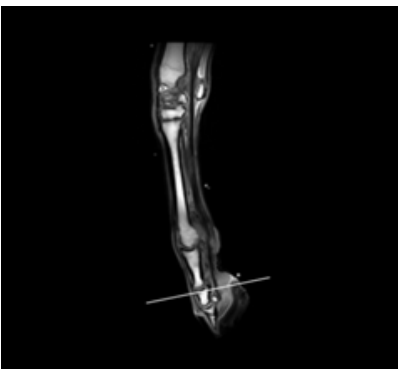

(e)

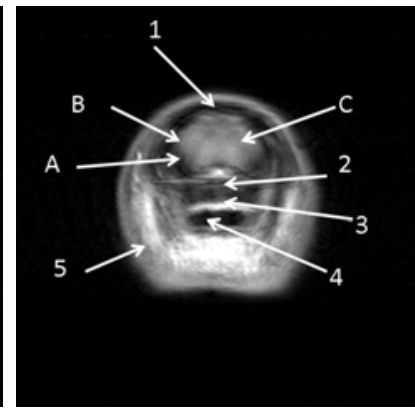

(c)

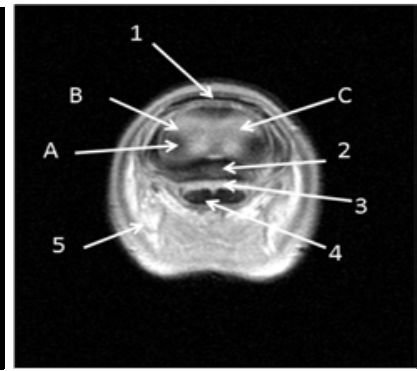

(f)

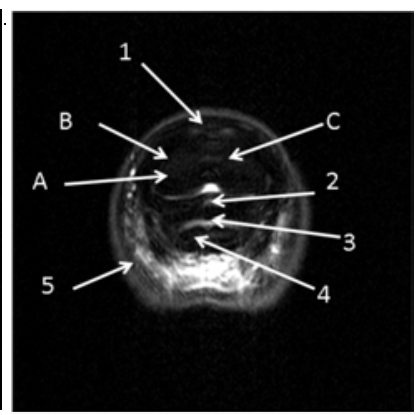

Fig. 11: a) Gross anatomic slice of hoof; b) the position of slice in the forelimb; c) T1-weighted image in transverse plane; d) T2-weighted image in transverse plane; e) PD image in transverse plane and f) STIR image in transverse plane. A: Long pastern bone; B: Pastern joint; C: Short pastern bone; 1: Common digital extensor tendon; 2: Oblique sesamoid ligament; 3: Straight sesamoid ligament; 4: Deep digital flexor tendon; 5: Lateral cartilage

(a)

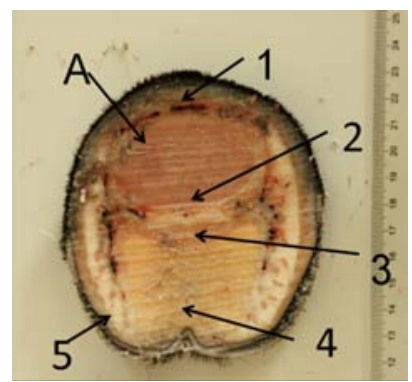

(d)

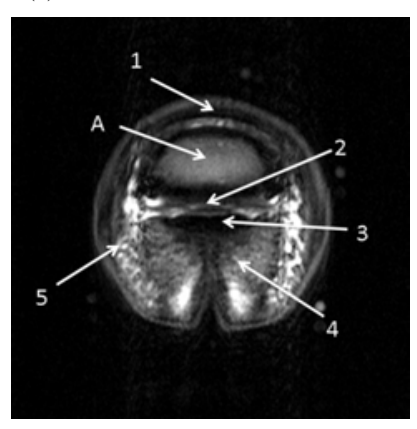

(b)

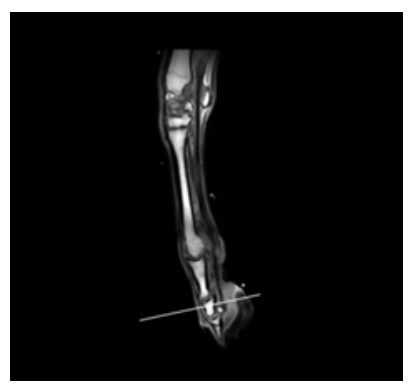

(e)

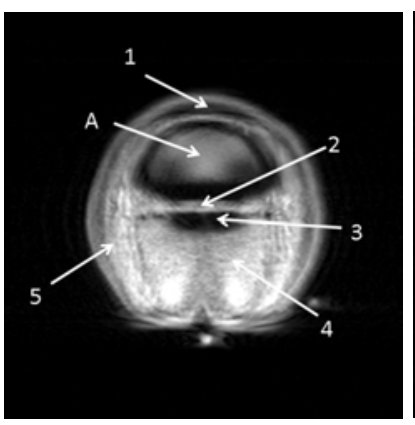

(c)

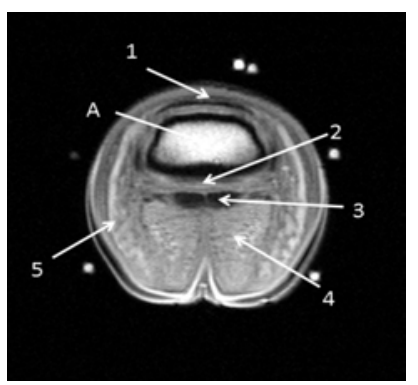

(f)

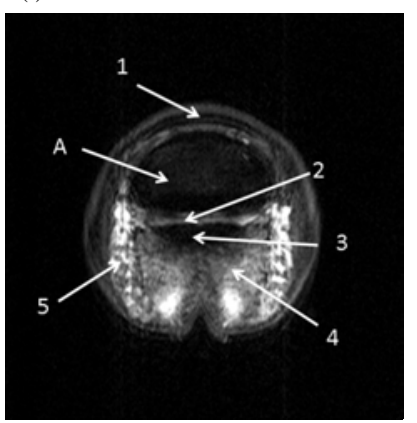

Fig. 12: a) Gross anatomic slice of hoof; b) the position of slice in the forelimb; c) T1-weighted image in transverse plane; d) T2-weighted image in transverse plane; e) PD image in transverse plane and f) STIR image in transverse plane. A: Short pastern bone; 1: Common digital extensor tendon; 2: Straight sesamoid ligament; 3: Deep digital flexor tendon; 4: Digital cushion; 5: Lateral cartilage 
In this study, the transverse plane images is chosen mainly based on two consideration. Firstly, as only one fore limb was studied during the slicing stage of the cadaver limb, it's imposible to make the 3-plane antomy slices simutanously and the transverse plane is the best choice on the safe side. Secondly, the axial image also havs its unique advantage. The lateral and medial tendons and ligaments were not clear on the sagittal images which are only fully displayed through the transverse images.

The horse studied in this study was a Chinese Mongolian yearling horse which is one of the most widely kept indigenous horse breeds of China. However, very few researches regarding the anatomical information imaged with MRI or other tomography were found. This fact might be attributable to three reasons: horse racing is yet to be a popular sport in China and the industry of sporting horse care has not gain as much attention as that in western countries.

A large scale modern veterinary medication system has not been established in China. High field MRI which has been widely adopted in veterinary medical examinations in Western countries is not practical to be applied in Chinese rural areas at current stage based on its high cost and maintenance standards. However, with the rapid development of the economy and the growing popularity of the horse racing sport in China, it is reasonable to foresee that an improved racing horse care system and ancillary veterinary services are urgently required. In this situation, this study aims to provide a limitations for the research of the Chinese horse illness.

However, there are limitations regarding this research. Although, researchers obtained $\mathrm{PD}$ and $\mathrm{T} 1$ weighted images with a high SNR, the images of STIR and T2 sequences still bear a low SNR for the mid-field MRI which is due to the long TR used for T2 and the inversion strategy for STIR sequence. In STIR sequence, the spin echo sequence is completed by a previous $180^{\circ}$ inversion pulse. Fat has a short TI. Hence, by choosing a short TI of $140 \mathrm{msec}$, the fat signal can be suppressed which also would lead to a lower SNR for the whole image. On the contrary, signals from tissues with long T1 and T2 relaxation times such as edema and inflammatory tissue can be enhanced.

Another problem of this study is that only one healthy horse limb was studied. Compared with previous research, like Dyson et al. (2003) studied the MRI used for equine feet with 15 horses and Murray et al. (2004) studied the performance for MRI used in limbs: with 64 horses, researchers may need more objects to examine so that some accidental abnormalities could be identified.

\section{CONCLUSION}

In this study, a quadrature knee coil and a 0.4 Tesla mid-field MRI scanner were used to obtain the T1weighted, T2-weighted, PD and STIR magnetic resonance images of a forelimb on an equine cadaver. The bony structures and soft tissue structures were revealed clearly on the images. Correlations between the images and gross anatomic sections were made. The results indicate that mid-field MRI contains the comparative SNR of T1 and PD weighted image with high field MRI which promise the mid-field MRI a broader application for equine diagnosis with a lower price and maintenance fee. Besides, the successful implementation of STIR sequence may also provide a valuable tool for diagnosis for the lameness in equine clinical.

\section{RECOMMENDATIONS}

In the future study, with further research and experience, more subjects with lameness will be examined with a better-designed U-style mid-field MRI.

\section{REFERENCES}

Blaik, M.A., R.R. Hanson, S.A. Kincaid, J.T. Hathcock, J.A. Hudson and D.K. Baird, 2000. Low-field magnetic resonance imaging of the equine tarsus: Normal anatomy. Vet. Radiol. Ultrasound, 41: 131-141.

Branch, M.V., R.C. Murray, S.J. Dyson and A.E. Goodship, 2007. Magnetic resonance imaging of the equine tarsus. Clin. Tech. Equine Pract., 6: 96-102.

Buckwalter, K.A., 1996. Magnetic resonance imaging of the knee. Radiology, 3: 99-122.

Bureau, N.J., P.A. Kaplan and R.G. Dussault, 1995. MRI of the knee: A simplified approach. Curr. Probl. Diag. Radiol., 24: 4-49.

Choquet, P., A. Constantinesco and H. Sick, 2000. MRI of the equine digit with a dedicated low-field magnet. Vet. Rec., 146: 616-617.

Dyson, S. and R. Murray, 2007. Magnetic resonance imaging of the equine foot. Clin. Tech. Equine Pract., 6: 46-61.

Dyson, S., R. Murray, M. Schramme and M. Branch, 2003. Magnetic resonance imaging of the equine foot: 15 horses. Equine Vet. J., 35: 18-26.

Mair, T.S. and J. Kinns, 2005. Deep digital flexor tendonitis in the equine foot diagnosed by low-field magnetic resonance imaging in the standing patient: 18 cases. Vet. Radiol. Ultrasound, 46: 458-466.

Marti-Bonmati, L. and M. Kormano, 1997. MR equipment acquisition strategies: Low-field or high-field scanners. Eur. Radiol., 7: 263-268. 
Murray, R.C., B.L. Roberts, M.C. Schramme, S.J. Dyson and M. Branch, 2004. Quantitative evaluation of equine deep digital flexor tendon morphology using magnetic resonance imaging. Vet. Radiol. Ultrasound, 45: 103-111.

Murray, R.C., S. Dyson, M. Branch and M. Schramme, 2007. Validation of magnetic resonance imaging use in equine limbs. Clin. Tech. Equine Pract., 6: 26-36.

Parizel, P.M., H.A. Dijkstra, G.P. Geenen, P.A. Kint, R.J. Versteylen, P.J. Van Wiechen and A.M. De Schepper, 1995. Low-field versus high-field MR imaging of the knee: A comparison of signal behaviour and diagnostic performance. Eur. J. Radiol., 19: 132-138.
Rutt, B.K. and D.H. Lee, 1996. The impact of field strength on image quality in MRI. J. Magn. Reson. Imaging, 6: 57-62.

Straaten, G.O. and S.N. Collins, 1995. Magnetic resonance imaging of the equine stifle: Normal anatomy. Vet. Radiol. Ultrasound, 36: 119-125.

Whitton, R.C., C. Buckley, T. Donovan, A.D. Wales and R. Dennis, 1998. The diagnosis of lameness associated with distal limb pathology in a horse: A comparison of radiography, computed tomography and magnetic resonance imaging. Vet. J., 155: 223-229.

Widmer, W.R., K.A. Buckwalter, M.A. Hill, J.F. Fessler and S. Ivancevich, 1999. A technique for magnetic resonance imaging of equine cadaver specimens. Vet. Radiol. Ultrasound, 40: 10-14. 